\title{
ESTIMATING THE IMPACT OF DESIGN AUTOMATION: THE INFLUENCE OF KNOWLEDGE ON POTENTIAL ESTIMATION
}

\author{
Rigger, Eugen (1,2); Lutz, Alexander (2); Shea, Kristina (2); Stankovic, Tino (2) \\ 1: V-Research GmbH; 2: ETH Zurich, Engineering Design and Computing Laboratory
}

\begin{abstract}
Assessing the impact of design automation on design practice prior to its implementation is difficult and subject to uncertainties. One reason for this is the designers' lack of knowledge about design automation. In this work, an industrial case study focusing on conceptual design of hydraulic circuits is conducted to assess the impact of the designers' knowledge on design automation potential estimation. In particular, the impact of demonstrating a prototypical implementation of a design automation application is investigated as a means to enhance the designers' knowledge about design automation. In this respect, a given set of metrics is rated twice to enable a comparative study: prior to and after introducing the design automation prototype. The yielded results show that the knowledge impacts the rating and supports reliability of potential estimation. Further, it is shown that designers acknowledge design automation potential for the early stages of design given sufficient knowledge about design automation. Yet, the results also indicate that careful attention needs to be put on the aspects covered by the prototype in order to avoid biasing participants.
\end{abstract}

Keywords: Conceptual design, Computational design methods, Case study, Design performance, Design automation

Contact:

Rigger, Eugen

V-Research GmbH

Design Automation

Austria

eugen.rigger@v-research.at

Cite this article: Rigger, E., Lutz, A., Shea, K., Stankovic, T. (2019) 'Estimating the Impact of Design Automation: The Influence of Knowledge on Potential Estimation', in Proceedings of the 22nd International Conference on Engineering Design (ICED19), Delft, The Netherlands, 5-8 August 2019. DOI:10.1017/dsi.2019.200 


\section{INTRODUCTION}

There is a substantial shortcoming of systematic assessment of the impact of design automation on design practice prior to its implementation (Verhagen et al., 2015). In this context, design automation refers to the application of computational methods and tools to support various aspects of the design process by automating design tasks. This enables to save time, generate alternative design solutions, explore solution spaces and reuse engineering knowledge. Current practice for potential estimation of design automation, which accounts for the assessment of the impact of design automation on design performance, relies on qualitative estimation of the value of design automation based on high-level design automation drivers. These drivers account for motivational aspects for design automation implementation in practice (Amen et al., 1999; Cederfeldt et al., 2005; Rigger and Vosgien, 2018) and most often, only estimations of potential time savings for repetitive tasks in product development are reported, e.g. Frank et al. (2014). Hence, quantifiable metrics are desirable to enable stakeholders to comprehensively understand the value of design automation application. However, even if a set of metrics is available, metrics need to be rated based on estimations of the impact. Since the majority of designers have no experience with design automation (Rigger and Vosgien, 2018), this estimation is subject to uncertainties.

The purpose of this paper is to systematically investigate these uncertainties when estimating the impact of design automation on design performance based on a prevalent set of metrics. To accomplish this, an industrial case study addressing the conceptual design of hydraulic circuits is set up in which the impact of the designers' knowledge of design automation on the related estimation of the potential value of design automation is assessed. In this context, a prototypical implementation of a design automation application is used to demonstrate the capabilities and modes of operation of design automation. By comparing the rating of metrics for quantification of the potential before and after introducing the design automation prototype, a comparative study is enabled.

The contributions of this paper are twofold: first, the impact of the knowledge of design automation on design automation potential estimation is assessed. Second, the yielded results highlight design automation potential for the early stages of the design process.

The paper is structured as follows: in Section 2, the relevant background on design automation potential estimation is critically reviewed. Section 3 introduces the research methodology regarding this work the details of the industrial use case and the experimental setup including workshop systematics and details about the participants. Following this, Section 4 presents the results that are critically discussed and analysed within Section 5. The paper closes with concluding remarks in Section 6.

\section{BACKGROUND}

In this section, the relevant work on the estimation of the impact of design automation on design practice is reviewed. Methods for potential estimation are introduced and critically assessed with respect to the underlying systematics for reliable and valid quantification of potential implications of design automation application on design performance. The section closes with concluding remarks regarding relevant work with implications for this paper.

\subsection{Methods for estimation of design automation potential}

Best practice for potential estimation of design automation aim at justification of design automation implementation based on the routine character of a design task (Emberey et al., 2007). Further, time savings and Return on Investment (ROI) estimations that are based on complexity assessment of a potential design automation solutions are proposed (van der Velden et al., 2012). However, these approaches do not provide the necessary details on estimation of implementation efforts. Addressing these shortcomings, Mulder et al. (2015) present an approach which differentiates between the type of design activities the automation is implemented for and the existing and desired levels of automation. A classification of activities as well as a listing of different levels of automation that can be attained for each activity is proposed. Based thereon, a cost coefficient value for each activity and level of automation is empirically determined. In combination with discrete event simulation of the design process and multi-objective optimization to investigate different levels of automation, a Pareto front with respect to lead time and investment cost is determined for various automation scenarios. In a 
similar manner, Pal and Ghosh (2017) assess efforts for software implementation in design and manufacturing processes based on the use case points method and related complexity assessment. Complexity of processes is assessed with respect to actors/interfaces, e.g. human-computer, systemsystem, etc., and the number of transaction for each use case involving interactions between an actor and a systems or design activities. These assessments in conjunction with weighting factors permit to estimate the effort for implementation of software tools based on the hours needed for implementation per use-case point. Despite being more systematic, the approaches by Pal and Ghosh (2017) and Mulder et al. (2015) strongly rely on empirical historical data for estimation of durations of design activities and implementation efforts. This makes it difficult to make reliable effort estimations due to the diversity of design automation tasks (Rigger et al., 2018). Further, in the work by Pal and Ghosh (2017) and Mulder et al. (2015), focus is put on the isolated assessment of the design automation task and related time savings for ROI analysis, rather than its integration in the design process and respective impacts on design performance in general.

Taking into account the design process perspective, Schut, Kosman, and Curran (2013) define highlevels objectives regarding cost, time and quality and related Key Performance Indicators (KPI) based on interviews with engineers. Following this, KPI measurements and process analysis with respect to value stream analysis are conducted to prioritize and refine the objectives. This prioritized list is then compared with initiatives addressing these objectives and corresponding effort estimations to enable informed decisions with respect to design automation implementation. Although the approach presents a valid systematic for justification of design automation implementation, it lacks details on identification of opportunities and definition of appropriate metrics for potential estimation. Building upon this approach, Verhagen et al. (2015) propose to analyse engineering processes with respect to information flows. Thereby, (design) automation potential is quantified based on assessment of information waste in product development. However, information waste is difficult to account for as the sole measure of efficiency in design, due to the iterative and situated character of design processes (Wynn and Clarkson, 2018).

\subsection{Implications for this work}

Only few methods exist addressing the potential estimation of design automation comprehensively. What is more, these methods usually do not investigate how to actually evaluate the metrics that reflect design practice and account for the design automation potential. Yet, this is necessary for informed decision about the impact of design automation implementation on design practice. Hence, there is a need to systematically investigate the evaluation of metrics prior to the implementation of design automation in practice.

\section{RESEARCH METHODOLOGY}

In order to investigate the impact of uncertainties in estimation of metrics values, the following section presents the applied research methodology. First, the impact diagram is derived to identify the influencing factors and isolate the one that is investigated in this study, namely the designers' knowledge of design automation. Following this, the method to conduct the study is detailed and the industrial use case including the background of the workshop participants is introduced. Finally, the experimental setup of the study is presented with a detailed description of the workshop systematic.

\subsection{Impact diagram \& applied method}

Figure 1 lists the influencing factors for design automation potential estimation determined based on assumptions and experience. The hypothesis of this work is that the knowledge about design automation impacts the rating of metrics. The impact of knowledge about design automation is of particular relevance, since the majority of designers have little or no knowledge of design automation especially considering the range of design tasks for which design automation can be applied for (Rigger et al., 2018). When the influencing factors related to the characteristics of participants (years of experience, role etc.) or the applied workshop systematic (w/o team discussions, applied rating scheme etc.) remain unchanged, the impact of knowledge on metrics rating for potential estimation of design automation can be assessed. Therefore, a comparative study (Blessing and Chakrabarti, 2009) is conducted comparing the rating of metrics before and after the participants are exposed to design 
automation prototype. Consequently, the participants act as both control and experimental group for conducting the experiment. In this work, a prototypical implementation of a design automation application tailored to the investigated use case is applied to demonstrate the capabilities and modes of operation of design automation and thereby improve the participants' knowledge of design automation.

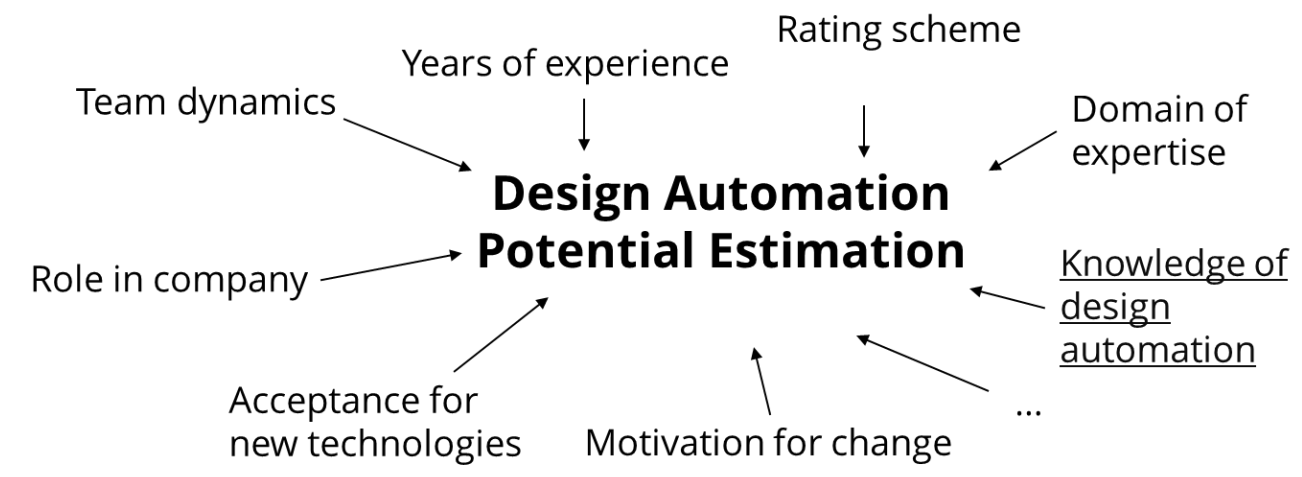

Figure 1: Impact diagram for potential estimation of design automation highlighting the influential factor assessed in the experiment.

\subsection{Industrial use case}

The industrial case focuses on design of hydraulic systems for heavy construction machinery and is conducted with a multinational company located in Austria. The design of hydraulic systems includes selection of concepts for realizing the hydraulic circuits for a given set of requirements and boundary conditions as well as selecting appropriate components and parameters. Thus, the design space is large and involves design activities that require creativity and design experience to identify appropriate solutions, in particular, for concept identification.

In total four designers participated in the study. The details of their position in the company and work experience can be found in Table 1.

Table 1: Workshop participants

\begin{tabular}{|l|l|l|}
\hline Participant & Position & Years of work experience \\
\hline Designer A & Designer, Team leader & 11 \\
\hline Designer B & Designer, Team leader & 11 \\
\hline Designer C & Department head & 35 \\
\hline Designer D & Designer & 4 \\
\hline
\end{tabular}

The case study for investigating potential estimation of design automation builds upon the results of preliminary studies that aimed at identification of opportunities for design automation application, and derivation of metrics for assessing the impact of design automation on design practice.

In particular, a predefined set of metrics for estimating the impact of "automated concept generation" on design practice is used in this work, see Table 3. Thereby, "automated concept generation" refers to the generation of hydraulic circuit topologies for given boundary conditions. Additionally, to illustrate a basic application of design automation as commonly applied in industry (Arora, 2004), a design automation prototype for "automated component selection" of closed-loop hydraulic circuits is implemented. Therefore, a mixed-integer nonlinear derivative-free algorithm is implemented yielding one cost-optimized solution given the design requirements and the constraints of the system. With respect to the "automated concept generation", the prototype is implemented based on the design automation method presented by Münzer and Shea (2017). For given boundary conditions, this prototype features automated generation of hydraulic circuits based on first order logic and Boolean satisfiability. Thus, multiple solutions can be generated for given boundary conditions. To illustrate the concept of automated validation of designs, simulations for representative architectures are (manually) created based on existing simulation libraries. As shown by Münzer and Shea (2017), this could potentially also be done automatically. 


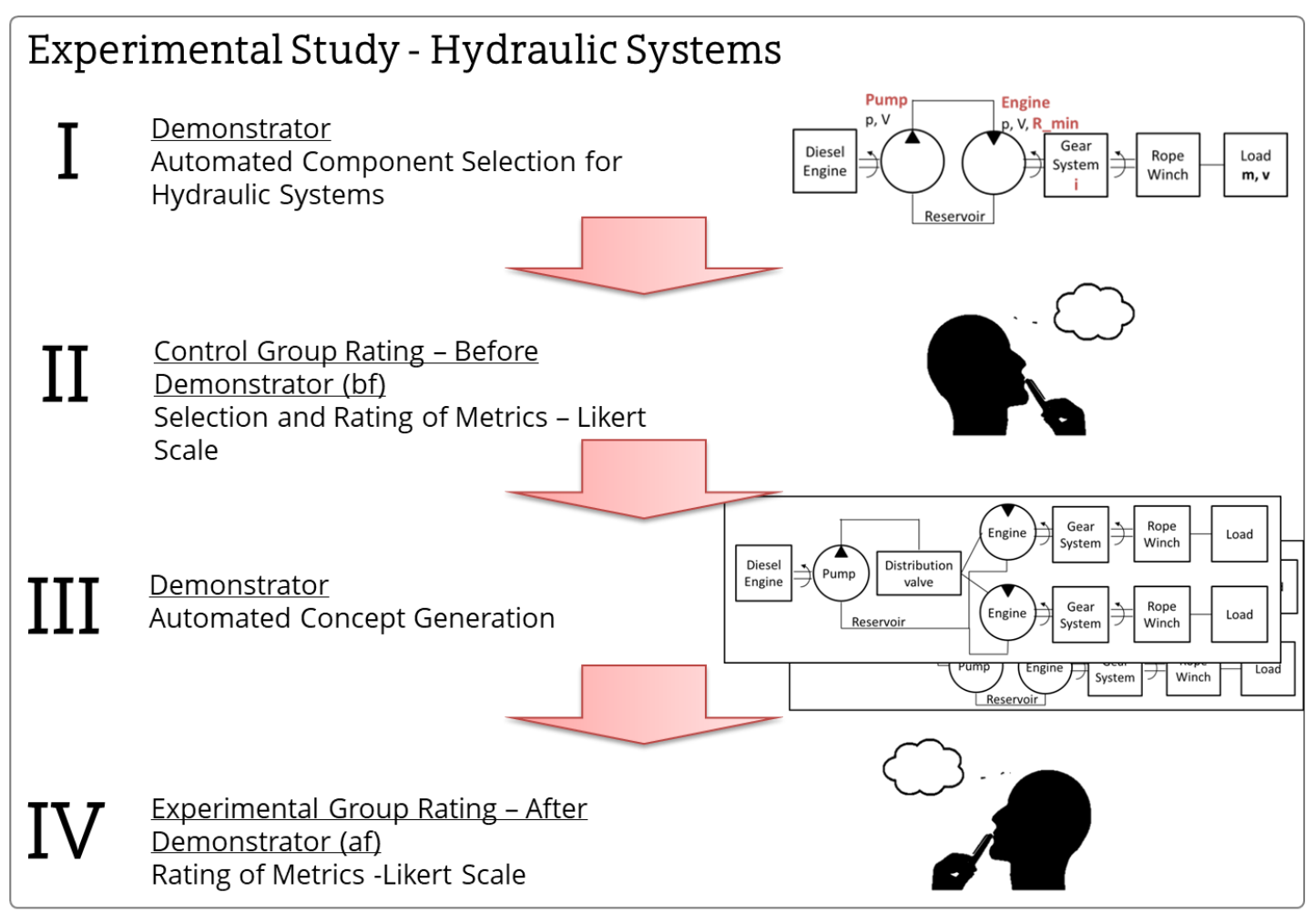

Figure 2: Applied systematic for conducting the comparative study

\subsection{Experimental setup}

In this section, the detailed procedure of the experiment is presented as shown in Figure 2.

First, the design automation prototype for automated component selection based on mixed-integer nonlinear derivative-free optimization was presented to illustrate a basic application of design automation. Following this, the workshop for selection and rating of metrics was conducted. This rating refers to the control experiments before showing the prototype for "automated concept generation" since the participants have not had any knowledge about this type of design automation prior to the demonstration. To realize the workshop, a printout of the list of metrics was provided to each participant. As illustrated in Table 3, the metrics account for the design automation task "automated concept generation". The metrics are rated on an individual basis for each participant without any interaction among the participants despite sitting in the same room doing the rating simultaneously.

After the first rating of metrics, the list of rated metrics is collected. Following this, the design automation prototype for automated generation of hydraulic concepts for given boundary conditions is presented. First, the aim of the design automation tool as well as the related task formalization such as inputs and outputs are explained in detail. Next, the different steps of the design automation method are presented as well as some intermediate results, e.g. generated product architecture as well as two representative related simulations. By doing so, the opportunity to generate multiple feasible solutions within a short amount of time is highlighted as well as the requirements and related efforts for design automation task formalization. To assess the impact of the change of knowledge of design automation, a new (blank) copy of the same list of metrics is provided for the participants. Due to the limited availability of data for metrics evaluation and for the sake of efficiency, qualitative rating of changes on metrics are indicated based on the Likert scale (Likert, 1932). Thereby, the Likert scale considering this study is defined as indicated in Table 2 .

Table 2: Likert scale applied for indication of quantitative changes of metrics due to design automation implementation

\begin{tabular}{|l|l|l|l|l|}
\hline-- & - & 0 & + & ++ \\
\hline $\begin{array}{l}\text { Strong decrease } \\
\text { of metric value }\end{array}$ & $\begin{array}{l}\text { Decrease of } \\
\text { metric value }\end{array}$ & No impact & $\begin{array}{l}\text { Increase of metric } \\
\text { value }\end{array}$ & $\begin{array}{l}\text { Strong increase } \\
\text { of metric value }\end{array}$ \\
\hline
\end{tabular}




\section{RESULTS}

Table 3 shows the evaluation of metrics values describing the impact of "automated concept generation" on design practice for design of hydraulic systems. For each metric the individual rankings of the four participants before and after viewing a design automation prototype for generation of hydraulic concepts is presented. The mean, standard deviation and range of responses are listed for each metric to indicate the difference in evaluations provided by the participants. This information is provided for each metrics twice, once before and once after the demonstration of the prototype. The metrics are layered with green colour if the change in the metrics value is considered beneficial and red for the case that metrics rating is disadvantageous. For the case that the metrics rating did not experience a change larger than 0.25 in mean, the background colour is white. With respect to this, Figure 3 shows a histogram highlighting how the rating of metrics varied due to the demonstration. The histogram further differentiates the results for each designer. Since the participants asked for anonymous review of results, the results cannot be mapped to the position and experience of participants. Nevertheless, the results permit deduction of the impact of showing a design automation prototype to designers that don't have any knowledge on the specific type of design automation task for which design automation potential should be estimated. To further illustrate, Figure 4 shows the impact of the metrics rating for a selected set of metrics. In particular, the rating of the metrics that experienced the strongest change of mean values due to introducing the design automation prototype is shown. Further, the rating of the ROI is listed as an example of a metric that did not change.

Table 3: Qualitative rating of metrics based on Likert scale for Case 2 before (bf) and after (af) presenting a design automation prototype for generation of hydraulic concepts

\begin{tabular}{|c|c|c|c|c|c|c|c|c|c|}
\hline \multirow{2}{*}{ 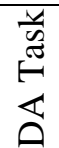 } & \multirow{2}{*}{\multicolumn{2}{|c|}{ Metric }} & \multicolumn{4}{|c|}{ Designer } & \multirow{2}{*}{$\sum_{\Sigma}^{\tilde{\Xi}}$} & \multirow{2}{*}{$\frac{\vec{D}}{\frac{1}{\Delta}}$} & \multirow{2}{*}{$\begin{array}{l}\infty \\
\ddot{\Xi} \\
\check{\Xi}\end{array}$} \\
\hline & & & 1 & 2 & 3 & 4 & & & \\
\hline & \multirow{2}{*}{$\begin{array}{l}\# \text { of concepts discussed in team / \# of } \\
\text { concepts discussed on average }\end{array}$} & bf & 0 & - & - & - & -0.75 & 0.43 & 1 \\
\hline & & af & + & + & + & ++ & 1.25 & 0.43 & 1 \\
\hline & \multirow[t]{2}{*}{ \# of stored designs / \# of designs created } & bf & ++ & + & + & + & 1.25 & 0.43 & 1 \\
\hline & & af & + & ++ & + & + & 1.25 & 0.43 & 1 \\
\hline & \multirow{2}{*}{$\begin{array}{l}\# \text { of product failure modes of products } \\
\text { delivered within specific duration / \# of } \\
\text { delivered products in specific duration }\end{array}$} & bf & 0 & ++ & + & 0 & 0.75 & 0.83 & 2 \\
\hline & & af & + & 0 & + & & 0.67 & 0.47 & 1 \\
\hline & \multirow{2}{*}{$\begin{array}{l}\text { Ideation Quality: Measures the feasibility of } \\
\text { an idea and whether it meets the design } \\
\text { requirements }\end{array}$} & bf & ++ & 0 & + & ++ & 1.25 & 0.83 & 2 \\
\hline & & af & 0 & + & + & 0 & 0.50 & 0.50 & 1 \\
\hline & \multirow{2}{*}{$\begin{array}{l}\# \text { of evaluated alternative designs per design } \\
\text { activity / average \# of solution alternatives } \\
\text { investigated per activity }\end{array}$} & bf & ++ & 0 & + & ++ & 1.25 & 0.83 & 2 \\
\hline & & af & + & 0 & + & ++ & 1.00 & 0.71 & 2 \\
\hline \multirow{9}{*}{ 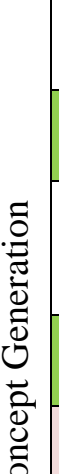 } & \multirow[t]{2}{*}{ Is the safety in the design process improved } & bf & ++ & + & ++ & ++ & 1.75 & 0.43 & 1 \\
\hline & & af & + & ++ & + & ++ & 1.50 & 0.50 & 1 \\
\hline & \multirow{2}{*}{$\begin{array}{l}\text { Time spent for scanning database for right } \\
\text { item / total activity time }\end{array}$} & bf & + & + & ++ & + & 1.25 & 0.43 & 1 \\
\hline & & af & - & + & ++ & + & 0.75 & 1.09 & 3 \\
\hline & \multirow{2}{*}{$\begin{array}{l}\text { Number of data items relevant to the user's } \\
\text { task and accessible / total number of data } \\
\text { items relevant to the user's task }\end{array}$} & bf & 0 & 0 & ++ & + & 0.75 & 0.83 & 2 \\
\hline & & af & + & - & ++ & ++ & 1.00 & 1.22 & 3 \\
\hline & \multirow{2}{*}{$\begin{array}{l}\text { Total expenditures per project / total average } \\
\text { cost per project }\end{array}$} & bf & + & + & ++ & + & 1.25 & 0.43 & 1 \\
\hline & & af & - & 0 & 0 & 0 & -0.25 & 0.43 & 1 \\
\hline & \multirow[t]{2}{*}{ Total time for conducting design task } & bf & ++ & + & ++ & + & 1.50 & 0.50 & 1 \\
\hline ¿̊ & & af & + & - & + & 0 & 0.25 & 0.83 & 2 \\
\hline \multirow{4}{*}{ 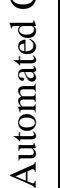 } & \multirow{2}{*}{$\begin{array}{l}\text { On-Time Delivery: Number of FCs released } \\
\text { on time / Total number of FCs }\end{array}$} & bf & + & 0 & 0 & 0 & 0.25 & 0.43 & 1 \\
\hline & & af & + & 0 & + & + & 0.75 & 0.43 & 1 \\
\hline & \multirow[t]{2}{*}{$\%$ of time spent for R\&D activities / project } & bf & - & + & + & + & 0.50 & 0.87 & 2 \\
\hline & & af & - & + & + & 0 & 0.25 & 0.83 & 2 \\
\hline
\end{tabular}


Table 4 continued.

\begin{tabular}{|c|c|c|c|c|c|c|c|c|c|}
\hline \multirow{2}{*}{ 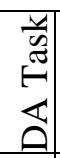 } & \multirow{2}{*}{\multicolumn{2}{|c|}{ Metric }} & \multicolumn{4}{|c|}{ Designer } & \multirow[b]{2}{*}{$\sum_{\Sigma}^{\mathbb{E}}$} & \multirow{2}{*}{ 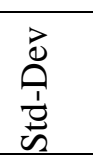 } & \multirow{2}{*}{$\begin{array}{l}\mathscr{\Xi} \\
\tilde{\Xi} \\
\check{\simeq}\end{array}$} \\
\hline & & & 1 & 2 & 3 & 4 & & & \\
\hline & \multirow{2}{*}{$\begin{array}{l}\text { \% of relevant ideation activities regarding } \\
\text { product, process and other domains by any } \\
\text { individual }\end{array}$} & bf & 0 & + & 0 & 0 & 0.25 & 0.43 & 1 \\
\hline & & af & - & + & + & + & 0.50 & 0.87 & 2 \\
\hline & \multirow[t]{2}{*}{\begin{tabular}{|l|} 
Employee satisfaction level \\
\end{tabular}} & $\mathrm{bf}$ & + & 0 & 0 & 0 & 0.25 & 0.43 & 1 \\
\hline & & af & 0 & - & 0 & 0 & -0.25 & 0.43 & 1 \\
\hline & \multirow{2}{*}{$\begin{array}{l}\text { Delay times: Mean and deviation, or } \\
\text { distribution, of wait times (best) }\end{array}$} & bf & 0 & 0 & + & + & 0.50 & 0.50 & 1 \\
\hline & & af & 0 & - & + & + & 0.25 & 0.83 & 2 \\
\hline \multirow{4}{*}{ 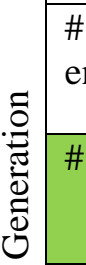 } & \multirow{2}{*}{$\begin{array}{l}\text { \# of engineering changes for specific design / } \\
\text { engineering changes for designs on average }\end{array}$} & bf & - & + & ++ & + & 0.75 & 1.09 & 3 \\
\hline & & af & - & + & ++ & 0 & 0.50 & 1.12 & 3 \\
\hline & \multirow[t]{2}{*}{ \# of rework rate due to defects in input } & bf & 0 & + & ++ & 0 & 0.75 & 0.83 & 2 \\
\hline & & af & - & 0 & 0 & + & 0.00 & 0.71 & 2 \\
\hline \multirow{6}{*}{ 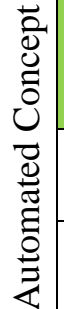 } & \multirow{2}{*}{$\begin{array}{l}\text { Key performance indicator of product / } \\
\text { performance according to specification }\end{array}$} & bf & 0 & + & 0 & + & 0.50 & 0.50 & 1 \\
\hline & & af & 0 & ++ & 0 & & 1.00 & 0.71 & 2 \\
\hline & \multirow{2}{*}{$\begin{array}{l}\text { ROI: yield of time savings / investment cost } \\
\text { for implementing DA }\end{array}$} & bf & + & - & + & 0 & 0.25 & 0.83 & 2 \\
\hline & & af & + & - & 0 & + & 0.25 & 0.83 & 2 \\
\hline & \multirow{2}{*}{$\begin{array}{l}\text { Personal Expenditures per project / average } \\
\text { personal cost per project }\end{array}$} & bf & 0 & - & 0 & + & 0.00 & 0.71 & 2 \\
\hline & & af & 0 & - & 0 & + & 0.00 & 0.71 & 2 \\
\hline
\end{tabular}

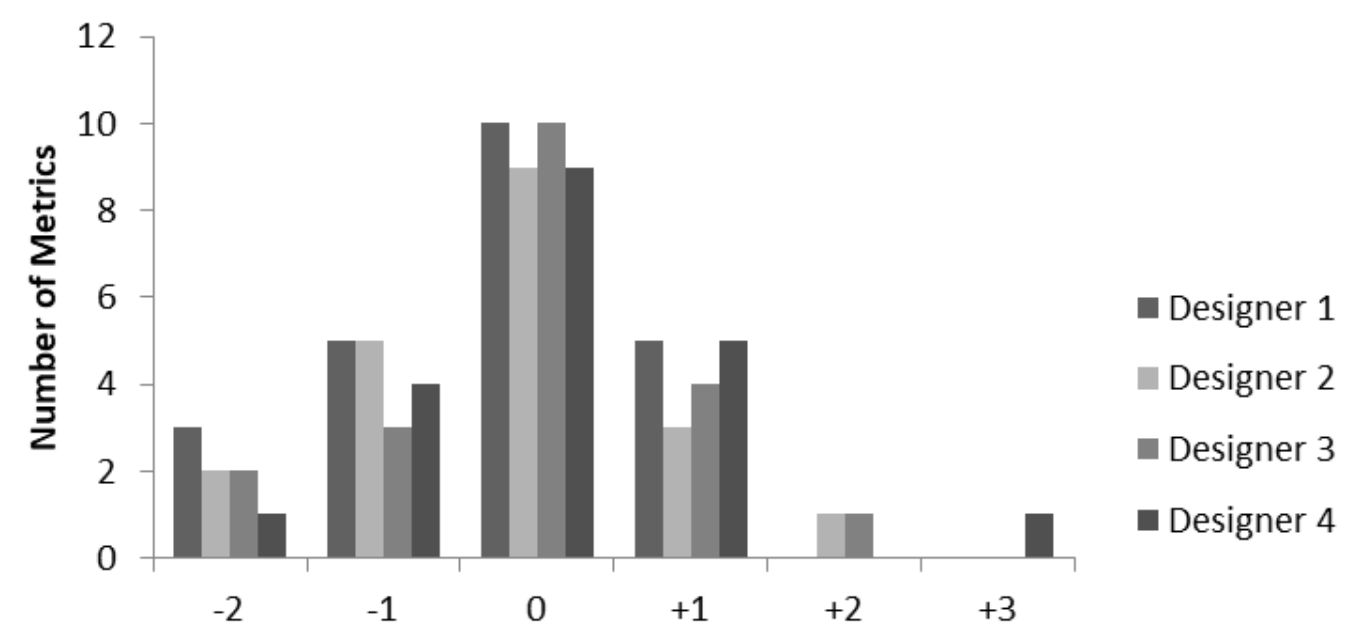

Cumulative difference in rating points after and before showing the prototype

Figure 3: Histogram indicating the impact of the prototype demonstration on rating of metrics for each designer

\section{DIscussion}

In this section, the impact of knowledge of design automation on potential estimation of design automation is critically assessed. Following this, the metric evaluation procedure is discussed, in particular the applied rating scale. Finally, implications of this research on design research and practice are highlighted, the limitations are indicated and future work is outlined. 


\subsection{Impact of knowledge of design automation}

As can be seen within Table 3 and Figure 3, showing a design automation prototype to designers that are not knowledgeable about design automation impacts the estimation of design automation potential. Figure 3 shows that the average rating of metrics slightly decreased. Since the Likert scale refers to the quantitative impact of design automation implementation on a metric, this does not necessarily mean that the overall potential for design automation implementation decreases. For some metrics an increase in the metric (e.g. Key performance indicator of product) is desirable and for some a decrease is beneficial (e.g. total time for conducting a task). Instead, a more realistic estimation of potential impacts of design automation implementation is enabled due to the gained knowledge. For example, the designers stated: "This is a powerful and desirable tool. Yet, this requires some effort to be able to account for the majority of cases. One person needs to permanently work with it". Based on Figure 4, this statement can be validated, since key performance indicators of the product are expected to increase while the expenditures and time for conducting a project decrease. Yet, the ROI remains the same, which is an indicator for the expected efforts for the maintenance of the design automation application.

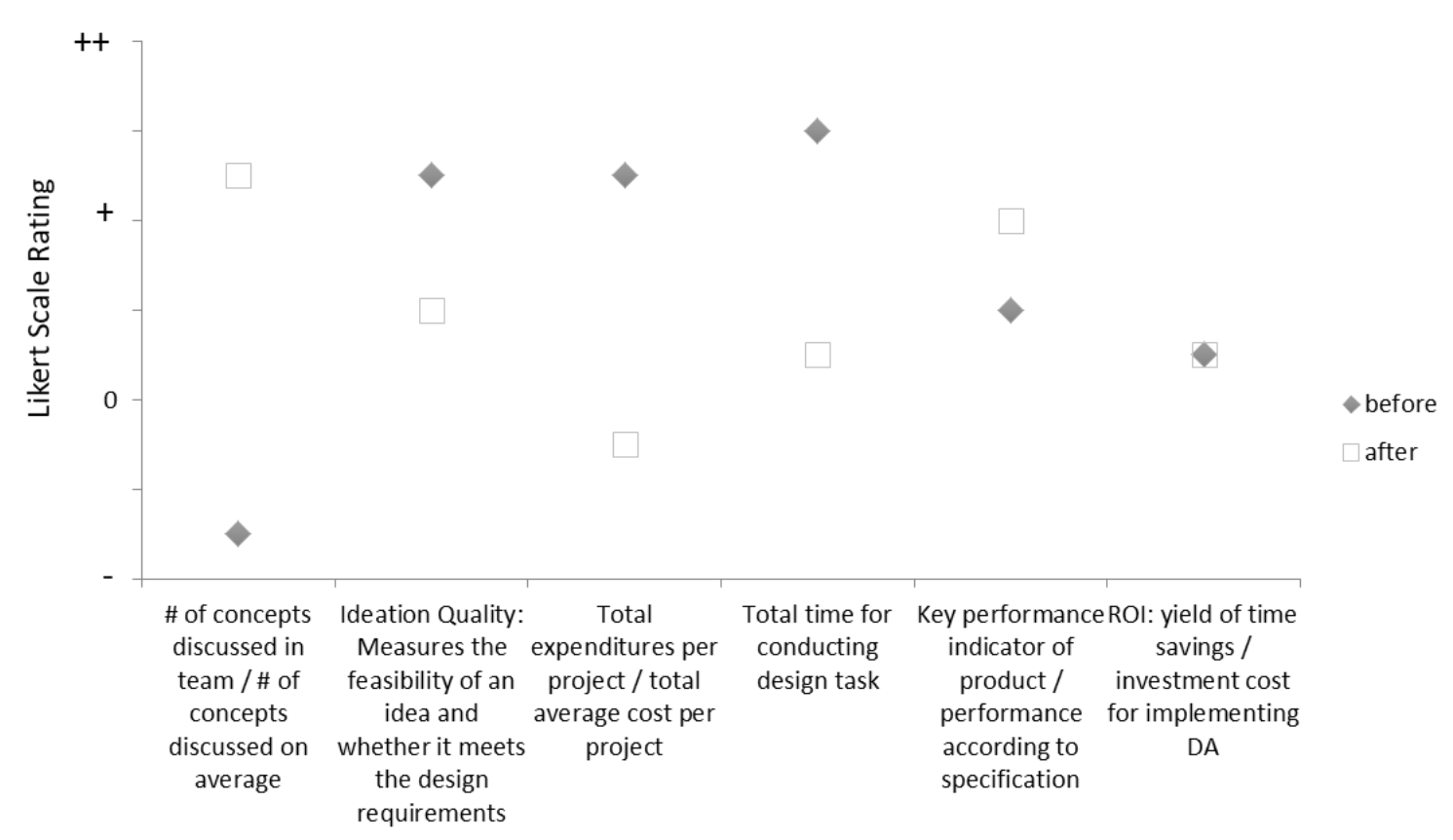

Figure 4: Direct comparison of rating of selected metrics that experienced the strongest change due to the demonstration of design automation prototype. ROI is listed as an example of a metric that did not change.

Further, Figure 3 shows that the majority of ratings are not strongly impacted by the design automation prototype. This leads to the assumption that the design automation prototype does not account for all the aspects covered by the metrics. As a result, careful attention needs to be paid not only to the selection of metrics but also to the features shown in the demonstration scenario. For example, total expenditures per project are considered to decrease. Yet, neither the list of metrics nor the demonstration accounts for the calculation costs that are not negligible for that type of automation. To equalize this impact, it is required to present the design automation prototype from different perspectives and that the related metrics are carefully selected. Preferably, the comprehensive list of metrics is gradually discussed based on the design automation prototype and its characteristics to clearly indicate how a metric is impacted by design automation implementation. If not, a design automation prototype biases participants and only partially impacts the rating of related metrics. To further elaborate on the integration of design automation prototypes for communication of the value of design automation, literature from related fields such as marketing research should be considered. For example, studies related to fit-risk that accounts for the customers' concern whether a technology really fits their needs (Parks et al., 2016) is to be considered.

Despite the challenges for implementing a design automation design automation prototype, designers consider it of fundamental support as remarked by one participant: "First it was very abstract. The design 
automation prototype made clear what we are actually talking about". Therefore, a basic knowledge is needed to be able to make informed decisions about design automation implementation and its benefits.

\subsection{Metric evaluation procedure}

For the experiment, the rating of metrics was conducted on an individual basis without any team discussions. This allows double-checking the reliability of metrics rating based on assessment of the agreement of results among the participants. Further, the Likert scale estimation was applied instead of numeric indication of differences for the sake of simplicity and efficiency of rating. Regarding reliability of rating, it can be seen within Table 3 that consistency of metrics among designers is in an acceptable range for the individual rating of designers. This is indicated by the standard deviation as well as the ranges of results that mostly span one or two classes of the Likert scale.

\subsection{Implications and future work}

The work presented in this paper shows that the knowledge on design automation is critical and strongly impacts the evaluation of metrics for potential estimation. Given the fact that designers are knowledgeable about design automation opportunities for the early phase, they also see the potential for design automation application as indicated by the metrics rating. This is contradictory to previous findings of survey that showed that designers do not consider design automation relevant for the early stages of the design process (Rigger and Vosgien, 2018). Most likely, this is linked to the lack of knowledge about the opportunities.

The work also highlights that design automation prototypes tailored to the investigated use case are a means to change the designers' perception of design automation. However, the development of the design automation prototypes requires considerable effort. Hence, future work needs to address the development of frameworks that facilitate design automation task formalization and enable rapid prototyping of design automation applications.

Regarding the evaluation of the metrics, the experiments were conducted with qualitative ratings based on the Likert scale. To increase expressiveness of the metrics rating, the future work needs to assess the application of a numeric scale to indicate changes in metrics. From a team dynamics point of view, it needs to be investigated how the rating of metrics is altered for the case that metrics are discussed in teams. Particular focus needs to be put on the hierarchies in teams that potentially lead to supressed opinions. Hence, additional case studies need to be conducted in future work to successively investigate these impacts.

Finally, it has to be noted that the work presented here does not provide external validity since only one experiment for once specific case is presented and the number of participants is strongly limited. Whereas the latter is difficult to increase when investigating design automation potential for specific design automation opportunities, external validity can be increased by conducting more experiments of the same type for different industrial use cases.

\section{CONCLUSIONS}

The work presented in this paper contributes by systematically assessing the influence of knowledge about design automation on the estimation of impact of design automation on design practice based on a case study conducted with an industry partner. With this respect, the impact of showing a prototypical implementation of a design automation application that is tailored to the use case of hydraulic concept design is evaluated as a means to increase reliability of potential estimation. The results indicate that the demonstration of the design automation prototype impacts the metrics rating and improves the designers' understanding of the underlying working principles of design automation. Thus, uncertainties due to a lack of knowledge of design automation are mitigated. Yet, careful attention needs to be put on the selection of metrics as well as the demonstration scenarios so as not to bias the designers. Finally, the results indicate the potential for design automation application in the early stages of design.

Future work needs to focus on conducting more experiments to increase external validity and investigation of other impacts such as team dynamics when rating the metrics or applied rating scales. 
Further, related work from other fields such as marketing research needs to be considered to improve knowledge about and the value of design automation.

\section{REFERENCES}

Amen, R., Rask, I. and Sunnersjö, S. (1999), "Matching Design Tasks to Knowledge-Based Software Tools When Intuition Does Not Suffice", Proceeding of DETC99, presented at the 1999 ASME Design Engineering Technical Conferences, Las Vegas, Nevada, pp. 1165-1174.

Arora, J. (2004), Introduction to Optimum Design, Academic Press, https://doi.org/10.1016/B978-0-12-064155$0 . X 5000-9$

Blessing, L.T.M. and Chakrabarti, A. (2009), DRM, a Design Research Methodology, Springer, Dordrecht; London, https://doi.org/10.1007/978-1-84882-587-1

Cederfeldt, M., Elgh, F. and others. (2005), "Design automation in SMEs-current state, potential, need and requirements", Proceedings of ICED 05, presented at the 15th International Conference on Engineering Design, Melbourne, Australia, 15.-18.08. 2005

Emberey, C.L., Milton, N., Berends, J.P.T.J., van Tooren, M.J.. and van der Elst, S.W. (2007), “Application of Knowledge Engineering Methodologies to Support Engineering Design Application Development in Aerospace", 7th AIAA Aviation Technology, Integration and Operations Conference (ATIO), Belfast, Northern Ireland. https://doi.org/10.2514/6.2007-7708

Frank, G., Entner, D., Prante, T., Khachatouri, V. and Schwarz, M. (2014), "Towards a Generic Framework of Engineering Design Automation for Creating Complex CAD Models", International Journal On Advances in Systems and Measurements, Vol. 7 No. 1 and 2, pp. 179-192.

Likert, R. (1932), "A technique for the measurement of attitudes", Archives of Psychology, Vol. 22 140, pp. 55-55.

Mulder, B., La Rocca, G., Schut, J. and Verhagen, Wim J, C. (2015), "A methodological approach for the optimisation of the product development process by the application of design automation", CEAS 2015, presented at the 5th CEAS Air and Space Conference "Challenges in European Aerospace, CEAS, Delft.

Münzer, C. and Shea, K. (2017), "Simulation-Based Computational Design Synthesis Using Automated Generation of Simulation Models From Concept Model Graphs", Journal of Mechanical Design, Vol. 139 No. 7, p. 071101. https://doi.org/10.1115/1.4036567

Pal, P. and Ghosh, K.K. (2017), "Estimating digitization efforts of complex product realization processes", The International Journal of Advanced Manufacturing Technology, https://doi.org/10.1007/s00170-017-1442-3

Parks, M., Bansal, S. and Zilberman, D. (2016), "Fit-risk in development projects: role of demonstration in technology adoption”, Environment and Development Economics, Vol. 21 No. 06, pp. 742-766. https://doi.org/10.1017/s1355770x16000188

Rigger, E., Stanković, T. and Shea, K. (2018), "Task Categorization for Identification of Design Automation Opportunities", Journal of Engineering Design, https://doi.org/10.1080/09544828.2018.1448927

Rigger, E. and Vosgien, T. (2018), "Design Automation State of Practice - Potential and Opportunities", Proceedings of the 15th International DESIGN Conference, presented at the DESIGN 2018, https://doi.org/10.21278/idc.2018.0537

Schut, E.J., Kosman, S. and Curran, R. (2013), “A Value Scan Methodology to Improve Industrial Operations", in Stjepandić, J., Rock, G. and Bil, C. (Eds.), Concurrent Engineering Approaches for Sustainable Product Development in a Multi-Disciplinary Environment, Springer London, London, pp. 411-423. https://doi.org/10.1007/978-1-4471-4426-7_36

van der Velden, C., Bil, C. and Xu, X. (2012), "Adaptable methodology for automation application development", Advanced Engineering Informatics, Vol. 26 No. 2, pp. 231-250, https://doi.org/10.1016/j.aei.2012.02.007

Verhagen, W.J.C., de Vrught, B., Schut, J. and Curran, R. (2015), "A method for identification of automation potential through modelling of engineering processes and quantification of information waste", Advanced Engineering Informatics, Vol. 29, pp. 307-321, https://doi.org/10.1016/j.aei.2015.03.003

Wynn, D.C. and Clarkson, P.J. (2018), "Process models in design and development", Research in Engineering Design, https://doi.org/10.1007/s00163-017-0262-7

\section{ACKNOWLEDGMENTS}

This work has been partially supported and funded by the Austrian Research Promotion Agency (FFG) via the "Austrian Competence Center for Digital Production" (CDP) under the contract number 854187. 